\title{
Refractory and Super-refractory Status Epilepticus
}

\author{
DEBOPAM SAMANTA ${ }^{1}$, LISA GARRITY ${ }^{2}$ AND RAVINDRAARYA ${ }^{2,3}$ \\ From ${ }^{1}$ Child Neurology Division, Department of Pediatrics, University of Arkansas for Medical Sciences, Little Rock, Arkansas; \\ ${ }^{2}$ Comprehensive Epilepsy Center, Division of Neurology, Cincinnati Children's Hospital Medical Center, Cincinnati, Ohio; and \\ ${ }^{3}$ Department of Pediatrics, University of Cincinnati College of Medicine, Cincinnati, Ohio; USA. \\ Correspondence to: Dr Ravindra Arya, Division of Neurology, Cincinnati Children's Hospital Medical Center, MLC 2015, 3333 \\ Burnet Avenue, Cincinnati, Ohio, 45229 USA.ravindra.arya@cchmc.org
}

\begin{abstract}
Context: Refractory status epilepticus (RSE) and super-refractory status epilepticus (SRSE) are neurological emergencies with considerable mortality and morbidity. In this paper, we provide an overview of causes, evaluation, treatment, and consequences of RSE and SRSE, reflecting the lack of high-quality evidence to inform therapeutic approach. Sources: This is a narrative review based on personal practice and experience. Nevertheless, we searched MEDLINE (using PubMed and OvidSP vendors) and Cochrane central register of controlled trials, using appropriate keywords to incorporate recent evidence. Results: Refractory status epilepticus is commonly defined as an acute convulsive seizure that fails to respond to two or more anti-seizure medications including at least one nonbenzodiazepine drug. Super-refractory status epilepticus is a status epilepticus that continues for $\geq 24$ hours despite anesthetic treatment, or recurs on an attempted wean of the anesthetic regimen. Both can occur in patients known to have epilepsy or de novo, with increasing recognition of autoimmune and genetic causes. Electroencephalography monitoring is essential to monitor treatment response in refractory/super-refractory status epilepticus, and to diagnose non-convulsive status epilepticus. The mainstay of treatment for these disorders includes anesthetic infusions, primarily midazolam, ketamine, and pentobarbital. Dietary, immunological, and surgical treatments are viable in selected patients. Management is challenging due to multiple acute complications and long-term adverse consequences. Conclusions: We have provided a synopsis of best practices for diagnosis and management of refractory/superrefractory status epilepticus and highlighted the lack of sufficient high-quality evidence to drive decision making, ending with a brief foray into avenues for future research.
\end{abstract}

Keywords: Convulsive status epilepticus, Epilepsy, Management, Outcome.

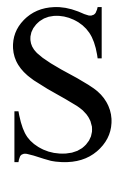
tatus epilepticus (SE) is a common pediatric neurological emergency. The definition of SE has evolved over time, to reconcile the likelihood of spontaneous seizure cessation based on pathophysiology, versus the operational urgency to achieve seizure termination and avoid adverse consequences. We have summarized some of the key definitions in Box I. The temporal evolution of SE is conventionally divided into early (5-30 $\mathrm{min})$, established (30-60 $\mathrm{min}$ ), and refractory ( $\geq 60 \mathrm{~min}$ ) phases [1].

There are two common definitions of refractory SE (RSE), which may operationally converge. These include a convulsive seizure lasting longer than 60 minutes, which may be continuous or intermittent without return to baseline mental status; and an acute convulsive seizure that fails to respond to $\geq 2$ anti-seizure medications (ASMs) including at least one non-benzodiazepine ASM. In our experience, the latter criteria is more commonly used in practice. At present, there is a dearth of sufficient high-quality evidence to formulate a uniform management strategy for RSE, resulting in variability in treatment approaches and the choice of therapeutic endpoint(s).
Partly because of this, about $15 \%-35 \%$ of RSE patients fail to achieve desired treatment response, and progress to super-refractory SE (SRSE), which is defined as an SE that continues for 24 hours or more despite anesthetic treatment, or recurs on an attempted wean of the anesthetic regimen [2,3].

We, herein, provide an overview of RSE and SRSE. However, we have not reviewed the epidemiology of RSE/ SRSE, because of insufficient data for these entities separate from SE. We have also not discussed the pathophysiology of drug-resistance in SE, because the relative importance of different molecular mechanisms remains uncertain at present, and does not inform treatment approach. Finally, our discussion is focused on RSE/SRSE evolving from a convulsive seizure with impaired awareness, and does not address epilepsia partialis continua.

\section{CAUSES AND RISK FACTORS}

The underlying causes of RSE/SRSE in a patient with no previous history of seizures, or possible triggers which may precipitate RSE/SRSE in a patient known to have 


\section{Box I Conceptual Evolution of the Definition of Status Epilepticus and Other Related Entities}

Conceptual basis for definition of status epilepticus

- Mechanistic: failure of homeostatic mechanisms to control a seizure.

- Physiological: ictal compromise of neuronal survival.

- Operational: ictal duration to warrant emergent treatment.

Definition of status epilepticus

- 1904: Seizures occur so frequently that coma and exhaustion are continuous between seizures. (Clark and Prout)

- 1940: Severest form of seizures in which the post-convulsive sleep of one attack is cut short by development of the next (seizure). (Wilson)

- 1964: Seizure persists for a sufficient length of time or is repeated frequently enough to produce a fixed and enduring epileptic condition. (ILAE $1^{\text {st }}$ International Classification of Epileptic Seizures)

- 1981: Seizure persists for a sufficient length of time or is repeated frequently enough that recovery between attacks does not occur. (ILAE revision)

- 1993: More than 30 minutes of (1) continuous seizure activity or (2) two or more sequential seizures without full recovery of consciousness between seizures. (AES working group on SE)

- 1998: Two or more generalized convulsions, without full recovery of consciousness between seizures, or continuous convulsive activity for more than 10 minutes (overt generalized convulsive SE). (Veterans Affairs Study)

- 1999: Operational definition: generalized convulsive SE in adults and children $>5$ years old refers to $\geq 5$ min of continuous seizures, or $\geq 2$ discrete seizures between which there is incomplete recovery of consciousness Mechanistic definition: failure of the "normal" factors that serve to terminate a typical generalized tonic-clonic seizure. (Lowenstein, Bleck, and Macdonald)

- 2015: A condition resulting either from the failure of the mechanisms responsible for seizure termination or from the initiation of mechanisms which lead to abnormally prolonged seizures (after time point $t_{1}$ ). It is a condition that can have long-term consequences (after time point $t_{2}$ ), including neuronal death, neuronal injury, and alteration of neuronal networks, depending on the type and duration of seizures. Where: for tonic-clonic SE ( $\left.t_{1}=5 \mathrm{~min}, t_{2}=30 \mathrm{~min}\right)$, focal SE with impaired consciousness $\left(t_{1}=10 \mathrm{~min}, t_{2}>60 \mathrm{~min}\right)$, and absence SE $\left(t_{1}=10-\right.$ 15 min, $t_{2}$ unknown) (ILAE Task Force on Classification of SE)

Other entities

- Refractory SE: SE that fails to respond to adequately used $1^{\text {st }}(\mathrm{BDZ})$ and $2^{\text {nd }}$ line (appropriately chosen nonBDZ) medications.

- Super-refractory SE: SE that persists for 24 hours or more after initiation of $3^{\text {rd }}$ line medications (anesthetics) or recurrence of seizure during withdrawal of the anesthetics.

- NORSE: New onset of refractory SE without a clear acute or active structural, toxic, or metabolic cause in a patient without active epilepsy or other preexisting relevant neurological disorder. It is a clinical presentation and not a specific diagnosis.

- FIRES: NORSE with a prior febrile infection between 2 weeks and 24 hours prior to onset of refractory SE, with or without fever at the actual onset of SE. It is a sub-category of NORSE.

AES: American Epilepsy Society; ILAE: International League Against Epilepsy; FIRES: Febrile Infection Related Encephalopathy Syndrome; NORSE: New-Onset Refractory Status Epilepticus; SE: Status Epilepticus].

epilepsy, are similar to those responsible for the more common convulsive SE. In general, any pathology which can trigger an acute symptomatic seizure can cause an SE which may progress to an RSE/SRSE. This includes neurological and systemic infections, acute vascular events, traumatic brain injury, and immune, metabolic, or toxic encephalopathies.

\section{Febrile SE: Lessons from FEBSTAT}

In the pediatric population, prolonged febrile seizures constitute the most common subgroup and account for up to $35 \%$ of all episodes of SE [4]. The importance of recognizing and promptly managing febrile SE has been underscored by the FEBSTAT study. In this study, 71\% of 
patients with acute changes on brain magnetic resonance imaging (MRI) after febrile SE, were found to have obvious hippocampal sclerosis 1 year later [5,6]. Given the importance of hippocampal sclerosis as a substrate for drug-resistant temporal lobe epilepsy, this finding emphasizes the direct association between the two. Another important finding from FEBSTAT study was the association of seizure duration with the time to initial treatment with benzodiazepines (BDZs), where every 2.7 min delay in the initial treatment was associated with 1.3 min increase in seizure duration [6,7]. Another study which found shorter median duration of febrile SE (35 min) compared to FEBSTAT ( $68 \mathrm{~min}$ ), potentially attributed it to shorter time to access emergency medical services and initial treatment [8].

\section{Infectious Causes}

The etiology of RSE/SRSE varies geographically, and studies from India have noted a predominance of infectious causes [9-11]. In a series of 148 adults with encephalitis, 18 were diagnosed with SE, predominantly in those with herpes simplex virus (HSV) infection or Japanese encephalitis [12]. Children were noted to be more susceptible to have encephalitis-related SE in this cohort. The diagnosis of HSV is important in SE/RSE patients, as treatment with acyclovir within 24 hours of onset has been shown to be associated with better prognosis [13]. A recent study reported convulsive SE in 41 patients with neurocysticercosis, although they did not progress to RSE/SRSE [14]. The duration of SE was found to be shorter in patients with single calcific lesion compared to those with degenerating cysts. In another study including 141 children presenting emergently with acute convulsive seizures, $49 \%$ were found to have neurocysticercosis, though again the progression to RSE was not reported [11]. There is a paucity of data on epidemiology of RSE/SRSE from India in other common infections including acute bacterial meningoencephalitis, cerebral malaria, and dengue. Differentiating acute symptomatic SE in a young child with a neurological infection, from a febrile SE, is a challenging and important consideration.

\section{Autoimmune and Genetic Etiologies}

Regarding RSE/SRSE, it is important to recognize two particular groups of diseases, which may prompt an etiology-specific treatment or prognostication early in the course of management. The first group includes autoimmune encephalitis. In children and adolescents, the leading autoimmune entity is anti-NMDA-receptor encephalitis, which can present with non-specific manifestations, such as, fever or headache in the prodromal stage. The next stage, involving the cerebral cortex, is the one which can present with RSE/SRSE in additional to behavioral symptoms. In children, these behavioral symptoms can take the form of new-onset temper tantrums in an otherwise well-adjusted child, unexplained episodes of aggression, and speech disturbances. Compared to the predominant temporal localization for seizures in adults with anti-NMDAR encephalitis, extra-temporal seizures, and sometimes even diffuse bilateral ictal onset can be seen in children, and should not exclude this consideration $[15,16]$. The other limbic encephalitis are also being increasingly recognized in children, and may often not occur as a para-neoplastic syndrome. In addition to seizures, which can progress to SE/RSE, other manifestations of limbic encephalitis can be relatively non-specific in children. The behavioral phenotype of limbic encephalitis in adults often consists of disturbances in recent memory, and affective symptoms including irritability, depression, or hallucinations. However, in children, non-specific disturbances of executive function (attention control, social inhibition, regulation of purposeful behavior) are often seen, and should be evaluated in a developmental context [17]. The second important etiologic subgroup of RSE/SRSE, which should be evaluated promptly, comprises of certain genetic epilepsies. These include, but are not limited to, Dravet syndrome and other sodium channelopathies, ring chromosome 20, pathogenic variants in polymerase-G or amino-acyl-tRNA synthetase genes affecting mitochondrial function, and Angelman syndrome [18].

\section{Seizure Triggers}

About 16-38\% SE episodes occur in children with a prior diagnosis of epilepsy, with low anti-seizure medication levels being the most common risk factor [4]. Low levels of regular anti-seizure medicines can, in turn, result from lack of adherence, scheduled withdrawal, insufficient dose, interaction with other concurrent medications, or growth spurt, etc. Other common precipitants of SE include intercurrent illness, exposure to a known trigger (e.g. sleep deprivation in some idiopathic generalized epilepsy syndromes), or metabolic decompensation.

\section{DIAGNOSTIC EVALUATION}

The goals of evaluation in RSE/SRSE include diagnostic verification, particularly in patients with subtle and nonconvulsive SE (NCSE); monitoring for therapeutic efficacy; diagnosis of the underlying etiology and/or risk factors; and early recognition of multisystem complications. We have summarized these in Table I, and briefly discuss the role of electroencephalography (EEG) and neuroimaging below.

\section{EEG Monitoring}

The foremost utility of EEG monitoring is to detect 
Table I Selected Investigations Useful in Management of Refractory and Super-refractory Status Epilepticus

Objective
Initial stabilization
Monitoring of seizure activity, and
titration for treatment response
Etiology of RSE/SRSE

Monitoring for treatment related complications

Monitoring for complications related to intensive care
Investigations

ABG, glucose, electrolytes, $\mathrm{CBC}$, liver and renal function tests

Electroencephalography

- Toxin screen

- Levels of anti-seizure medication

- Brain MRI (cerebrovascular diseases, tumors, malformations of cortical development, TBI, HIE, autoimmune encephalitis)

- Acquire MRS with MRI, plasma ammonia, lactate, pyruvate, acylcarnitine profile, plasma amino acids, urine organic acids (IEMs)

- CSF biochemistry, microscopy, culture, viral studies, neurotransmitter levels (CNS infections, neurotransmitter disorders, certain IEMs)

- Autoimmune encephalitis panel, CRP, ANA and other antibodies (Ro/SSA, La/SSB, Sm, RNP, dsDNA, anti-phospholipid, ANCA), C3, C4, serum cryoglobulins, serum immune electrophoresis, quantitative Ig levels, MR angiography (autoimmune encephalitis, vasculitis)

- Specialized muscle or liver tissue studies (suspected mitochondrial disorders)

- Chromosomal microarray and epilepsy next-generation sequencing panel (genetic causes)

- Brain biopsy (unknown cause)

Related to ASMs: ASM levels, serum albumin

Related to ketogenic diet: ABG, CBC, lipid profile, carnitine, ECG, gastro-intestinal motility studies, calcium, alkaline phosphatase, 25-OH-D levels, liver and renal function tests, levels of magnesium, selenium, copper, and zinc, coagulation screen, and serum beta-hydroxy butyrate levels for ketosis

- CXR (pulmonary edema, embolism, aspiration or ventilator-associated pneumonia)

- Brain MRI (hypoxic or vascular injury, cerebral edema)

- Biochemistry to monitor liver, kidney, and pancreatic function, and electrolyte levels

- Monitoring for disseminated coagulopathy, thrombophlebitis, rhabodomyolysis

- NCS/EMG (critical illness neuropathy)

- $\mathrm{CBC}$, appropriate cultures (iatrogenic infections/sepsis)

- Calcium, alkaline phosphatase, x-rays, DEXA (bone health and fractures)

ABG: arterial blood gases, ANA: antinuclear antibody, ASM: anti-seizure medication, CBC: complete blood count, CNS: central nervous system, CSF: cerebrospinal fluid, CXR: chest x-ray, ECG: electrocardiography, EEG: electroencephalography, EMG: electromyography, HIE: hypoxicischemic encephalopathy, IEM: inborn error of metabolism, MRI: magnetic resonance imaging, MRS: magnetic resonance spectroscopy, NCS: nerve conduction studies, TBI: traumatic brain injury.

Etiologies or complications related to a particular investigation are provided in parenthesis. This list is not exhaustive, and should be guided by clinical judgement.

ongoing subclinical seizure or NCSE after the convulsive seizure has apparently terminated. Hence, professional societies have recommended continuous EEG monitoring to be initiated within 1 hour of SE, and continued for at least 24-48 hours, often longer in the presence of altered consciousness [19-21]. Although the EEG diagnosis of NCSE has been fraught with disagreement, recent studies have shown good diagnostic validity and inter-rater agreement for the Salzburg criteria [22,23]. In patients without known epileptic encephalopathy, occurrence of $>2.5 \mathrm{~Hz}$ epileptiform dischargesmay be sufficient for the diagnosis of NCSE. In case of epileptiform discharges $\leq 2.5 \mathrm{~Hz}$, or the ictal pattern limited to rhythmic slow waves, it is important to evaluate for electroencephalographic improvement with a bolus of intravenous BDZ; associated subtle clinical manifestations; and typical spatiotemporal evolution in the morphology, frequency, or locus of the electroencephalographic pattern. The availability of synchronized video monitoring, allowing simultaneous review of video-EEG is important in such situations. The diagnosis of NCSE becomes more challenging in patients with a known epileptic encephalopathy. In such patients, diagnosis of NCSE requires evidence of a distinct increase in the locus, field, 
or frequency of epileptiform discharges, and an obvious change in clinical state, or response to an intravenous BDZ bolus [23]. Hence, in these patients, it is important to have a good understanding of the baseline EEG pattern prior to the onset of RSE, based on a personal review of previous EEGs.

Other periodic and rhythmic EEG patterns, which do not meet the criteria for NCSE, are often labelled as lying on an interictal-ictal continuum. Although, this terminology is an useful EEG descriptor, its clinical significance remains uncertain. However, recognition of periodic patterns including lateralized periodic discharges (LPDs) or generalized periodic discharges (GPDs) may be important because of some association with the risk of seizure recurrence and certain specific conditions, such as, LPDs with stroke and herpes encephalitis [24]. Rarely, other EEG patterns may suggest a specific etiology, for example, extreme delta brushes in anti-NMDAR encephalitis.

Use of quantitative EEG has been increasing recently in neuro-critical care practice, due to integration of analysis software into EEG reading suites [25]. During management of RSE/SRSE, quantitative analysis of EEG trends may be helpful in evaluation of the suppression ratios over the course of treatment, recognition of periodic patterns or seizure recurrence, and comparison of power ratios in canonical EEG frequency bands. However, there is a paucity of studies validating use of quantitative EEG for prediction of outcomes in RSE/SRSE. Hence, it is important to verify any observations on quantitative trends, with a careful review of raw EEG. In our practice, the two most common uses of quantitative EEG in the management of RSE/SRSE include temporal analysis of suppression ratios, and quantitation of seizure burden once the specific imprint of a given seizure type is recognized on the density spectral array or amplitude integration. A detailed discussion of the methods, utility, and pitfalls of quantitative EEG is outside the scope of this paper.

The second most important role of EEG monitoring in the management of RSE/SRSE is to establish the therapeutic target. In these children, clinical evaluation for continued seizure activity is often non-informative, and becomes more so as the anesthetic infusions are increased, or neuromuscular blockers are used to facilitate airway control. At this point, the EEG usually shows electrographic bursts interrupted by periods of relative diffuse amplitude suppression. The optimal EEG target for treatment of RSE/SRSE is not strictly defined, and have included, suppression of seizures, varying degrees of suppression-burst pattern, and nearly complete EEG suppression [26]. Our practice is to aim for complete suppression of seizures, and achieve $50 \%-70 \%$ suppression ratio. Once this therapeutic goal is achieved, EEG monitoring is continued for 24-48 hours, when attempts are first made to wean off the infusions. EEG monitoring is important at this point to recognize the occurrence of emergence seizures or bursts with epileptiform elements, though these are not always treated individually.

\section{Neuroimaging}

The primary goal of neuroimaging in RSE/SRSE is to help identify the etiology, which in some cases, may lead to a specific urgent intervention. Secondly, identification of a potentially epileptogenic lesion may prompt consideration of emergency epilepsy surgery for RSE/SRSE in centers where such expertise is available. However, the interpretation of MRI studies early in the course of an RSE/SRSE can be challenging due to seizure-related or treatment-related changes. Specifically, it may be worthwhile to distinguish vasogenic edema reflected by T2/FLAIR hyper-intensities, from cytotoxic edema predominantly manifested as diffusion restriction. This is because vasogenic edema can sometimes point to an underlying etiology, whereas cytotoxic edema often represents compromised neuronal integrity from RSE/ SRSE. In many patients there is an overlap between the two, and it is helpful to follow their neuroimaging over time. It is also important to look for confounding factors, for example, vigabatrin can be associated with both $\mathrm{T} 2$ hyper-intensities and restricted diffusion, predominantly in corpus callosum and deep gray structures [27,28]. In the FEBSTAT study, about $10 \%$ of children with a single febrile SE episode were found to have altered signal in the hippocampal region. After one year, $86 \%$ of them showed hippocampal volume loss and $71 \%$ had evidence of hippocampal sclerosis [5], emphasizing the need for longitudinal neuroimaging to ascertain neuronal injury in patients with RSE/SRSE.

\section{TREATMENT}

The primary goal of treatment is the termination of clinical and electrographic SE. However, the objectives of treatment in RSE/SRSE are somewhat different from those in early/established SE. In patients with early/established SE, the treatment efforts are focused on rapid seizure control to avoid neuronal injury. However, by definition, RSE represents a situation where pathophysiological mechanisms that support drug-response in established SE have been overwhelmed or altered, and hence, conventional treatment approaches are unlikely to be successful. Further, in SRSE, it is very likely that excitotoxic and other processes which compromise neuronal survival, 
have already been activated. Hence, the goal of treatment in RSE/SRSE is to limit or reverse these processes, prevent their downstream consequences, and salvage end-organ function to the maximum possible extent [29]. Clinically, this means that the treatment plan should encompass not only seizure control, but to also avoid, anticipate, and manage multisystem dysfunction resulting from ongoing seizure activity, from medications, and from prolonged unconsciousness and immobility [30]. While the intensive care related to RSE/SRSE is outside the scope of this paper, we have briefly summarized the first and second line treatment for SE, and then discussed specific therapeutic approaches to RSE/SRSE in more detail.

\section{First- and Second- line Drug Treatments}

The American Epilepsy Society guidelines published in 2016, have synthesized available evidence for drugtreatment of early and established SE [31]. There is consensus that BDZs represent the initial treatment of choice for SE, with equipoise among intramuscular midazolam (MDZ), intravenous lorazepam (LZP), and intravenous diazepam (DZP). This equipoise is based on three class I randomized controlled trials, which are summarized below. The Veterans Affairs study compared four interventions including DZP followed by phenytoin (PHT), LZP, phenobarbital (PHB), and PHT alone [32]. There were no significant differences among these 4 interventions on intention-to-treat (ITT) analysis. However, on pairwise comparisons in patients with generalized convulsive SE, LZP was superior to PHT, for cessation of all motor and EEG seizure activity within 20 min of starting drug infusion and no recurrence of seizure activity during the next $40 \mathrm{~min}$. The Rapid Anticonvulsant Medication Prior to Arrival Trial (RAMPART), showed equivalence between intramuscular MDZ and intravenous LZP for the proportion of patients who achieved seizure termination without need for additional rescue [33]. However, the auto-injectors used in RAMPART are not available commercially, and whether conventional intramuscular MDZ injection will have similar efficacy is open to question. The third class I trial, showed equivalence between intravenous LZP and DZP for the proportions of patients with seizure termination, need for assisted ventilation, recurrence within 1 hour, and time to SE termination [34].

Only when these three options are not available, or not feasible due to challenges in achieving intravenous access in a convulsing child, other treatments may be considered, including rectal DZP, intranasal MDZ, and buccal MDZ. A network meta-analysis of 16 studies, specifically looking at non-venous treatments for acute convulsive seizures, found intramuscular and intranasal MDZ to be superior to other comparators for clinically relevant outcomes [35]. Specifically, this meta-analysis excluded data from RAMPART in their quantitative synthesis, thus independently supporting the efficacy of conventional intramuscular MDZ and encouraging the use of intranasal MDZ. From a practical standpoint, MDZ has the advantage of not needing refrigeration for storage. In US, MDZ nasal spray has been recently approved to treat seizure clusters and acute repetitive seizures [36]. Intranasal formulations of DZP and rapid systemic delivery of alprazolam (staccato alprazolam) by inhalation of thermally generated aerosol from a single-use hand-held device, are currently undergoing evaluation [37,38].

Appropriate first-line drug-treatment results in seizure termination in about $65 \%$ of patients. However, the subsequent choice of treatment is not driven by rigorous evidence. Traditionally, intravenous PHT has been the most commonly used $2^{\text {nd }}$ line treatment for SE. Fosphenytoin (FOS) has been preferred over PHT, due to the ability for faster infusion, less risk of cardiac arrhythmias, and decreased incidence of local tissue reaction. However, a meta-analysis of 22 studies has found the efficacy of PHT to be below, though not significantly, to that of valproate (VPA), PHB, and levetiracetam (LEV) [39]. This meta-analysis also concluded that there is insufficient data for the use of lacosamide (LAC) in the treatment of SE. Recently, two different open-label trials: ConSEPT study from Australia and New Zealand, and EcLiPSE study from United Kingdom, have compared LEV and PHT in children with BDZ-refractory SE [40,41]. Clinical cessation of seizure activity 5 min after infusions were not statistically different between the two groups. Overall, $50 \%-70 \%$ of participants achieved primary efficacy endpoint. An ongoing trial in US, Established Status Epilepticus Treatment Trial (ESETT), is also comparing LEV, FOS, and VPA [42]. Given the lack of class I trials, decisions about $2^{\text {nd }}$ line treatment are often based on local availability, cost, and most importantly patientspecific factors.

In addition to medical stabilization, the treatment of early/established SE proceeds concurrently with attempts to ascertain the etiology of SE. The specific treatments are driven by presumptive or definite etiology, often determined by local epidemiology, and investigative resources. A few suggestions are offered in Table II.

\section{Third-line Treatment: Drug Therapy for RSE}

There is a further paucity of clinical trials for drug treatment of RSE. Designing comparative effectiveness trials in this population has been challenging due to several reasons, including heterogeneity of $1^{\text {st }}$ and $2^{\text {nd }}$ line treatment choices, concurrent use of multiple other 
Table II Suggested Etiology-Specific Treatments in Status Epilepticus

\begin{tabular}{|c|c|c|}
\hline Etiology & & Suggested treatment \\
\hline Metabolic disturbances & $\begin{array}{l}\text { Hypoglycemia } \\
\text { hyponatremia, hypocalcemia, } \\
\text { hypomagnesemia, other } \\
\text { electrolyte disturbances }\end{array}$ & $\begin{array}{l}\text { Appropriate correction with IV dextrose } \\
\text { Appropriate corrections }\end{array}$ \\
\hline \multirow[t]{3}{*}{$\begin{array}{l}\text { Inborn errors of metabolism } \\
\text { or genetic causes }\end{array}$} & $\begin{array}{l}\text { Alper's syndrome (POLG and } \\
\text { ARS variants) }\end{array}$ & Avoid valproic acid \\
\hline & Dravet's syndrome & Valproic acid preferred as $2^{\text {nd }}$ line treatment \\
\hline & Presumptive suspicion & Consider empiric trial of pyridoxine and/or folinic acid \\
\hline Traumatic brain injury & & $\begin{array}{l}\text { Identification and management of increased intracranial } \\
\text { pressure }\end{array}$ \\
\hline Febrile status epilepticus & & $\begin{array}{l}\text { Fever control and prompt identification of the underlying } \\
\text { cause of fever }\end{array}$ \\
\hline \multirow[t]{5}{*}{ Infections } & Herpes simplex virus encephalitis & Acyclovir \\
\hline & Neurocysticercosis & $\begin{array}{l}\text { Anti-parasitic and anti-inflammatory treatment tailored to } \\
\text { individual patient circumstances based on nature and } \\
\text { number of lesion(s) }\end{array}$ \\
\hline & Cerebral malaria & Artesunate \\
\hline & Acute bacterial meningitis & $\begin{array}{l}\text { Empirical treatment with a third-generation cephalosporin } \\
\text { with/without vancomycin }\end{array}$ \\
\hline & Bartonella encephalitis & $\begin{array}{l}\text { Usually doxycycline and rifampin, in children }<8 \text { year of age } \\
\text { doxycycline can be replaced with azithromycin or } \\
\text { trimethoprim-sulfamethoxazole }\end{array}$ \\
\hline Autoimmune encephalitis & & Immunotherapy \\
\hline Psychogenic status epilepticus & & Avoid treatment with anti-seizure medications \\
\hline Toxic & Theophylline ingestion & Phenytoin may be avoided \\
\hline
\end{tabular}

This list is not exhaustive and should be modified according to local epidemiology and available resources.

medications affecting the nervous system, variability in the time course of administration of different medications, and the level of supportive intensive care. This is exemplified by a multicenter trial which was terminated inconclusively, because only 14 patients could be recruited over 3 years, against an estimated sample size of 150 [43]. Another trial of brexanolone (an aqueous formulation of the neuroactive steroid allopregnanolone) in 25 patients with SRSE was also inconclusive regarding efficacy end point, though it showed reasonable tolerability [44].

Conventionally, the three most commonly used anesthetic agents for the treatment of RSE include MDZ, short-acting barbiturates (pentobarbital/thiopentone), and propofol (Table III). Currently, MDZ is perhaps the most commonly used drug for RSE due to faster onset of action and short duration of effect [45]. However, use of MDZ in RSE/SRSE is fraught with several issues including development of tolerance, prolonged half-life with continued use, and potential for interactions with other drugs, nephrotoxicity, hepatotoxicity, and cardio- respiratory depression $[45,46]$. Several studies have reported on the use of $\mathrm{MDZ}$ for RSE/SRSE, using different doses and treatment targets, as reviewed elsewhere [45]. A meta-analysis including 111 children showed that MDZ was as effective as other coma inducing medications, but had a lower mortality [47]. However, another study that compared MDZ and DZP in 40 children and found a similar efficacy $(86 \%$ and $89 \%$, respectively), reported MDZ to be associated with a higher recurrence $(57 \%$ vs $16 \%)$ and higher mortality (38\% vs 10.5\%) [48]. Seizure control has been reported as occurring within 0.3-1.1 hours [45]. Breakthrough seizures have been reported in $47-57 \%$ of patients [49].

To compare, barbiturates have been used for the longest period, and believed to have higher efficacy. A meta-analysis including 193 adults with RSE compared pentobarbital, MDZ, and propofol. Although pentobarbital was associated with a significantly lower incidence of short-term treatment failure, breakthrough seizures, and the need to change to a different medication, it was also associated with a significantly higher frequency 
Table III Continuous Infusions for Treatment of Refractory Status Epilepticus

\begin{tabular}{|c|c|c|c|c|c|c|}
\hline Medication & $\begin{array}{l}\text { Mechanism } \\
\text { of action }\end{array}$ & $\begin{array}{l}\text { Initial } \\
\text { dose }\end{array}$ & Titration & $\begin{array}{l}\text { Usual } \\
\text { dose }\end{array}$ & $\begin{array}{l}\text { Absolute maximum } \\
\text { rate or concentration }\end{array}$ & Comments \\
\hline Midazolam & $\begin{array}{l}\text { GABA } \\
\text { receptor } \\
\text { agonist }\end{array}$ & $\begin{array}{l}0.2 \mathrm{mg} / \mathrm{kg} \\
\text { bolus, then } \\
0.05-0.2 \\
\mathrm{mg} / \mathrm{kg} / \mathrm{h}\end{array}$ & $\begin{array}{l}\text { Bolus } 0.2 \mathrm{mg} / \mathrm{kg} \\
\text { up to } 10 \mathrm{mg} \text { and } \\
\text { increase by } 0.2 \mathrm{mg} / \\
\mathrm{kg} / \mathrm{h} \text { every } 15-20 \\
\text { min until burst } \\
\text { suppression }\end{array}$ & $\begin{array}{l}0.05-0.2 \\
\mathrm{mg} / \mathrm{kg} / \mathrm{h}\end{array}$ & $\begin{array}{l}3.3 \mathrm{mg} / \mathrm{kg} / \mathrm{h} \text { to } \max \\
\text { total dose of } 100 \\
\mathrm{mg} / \mathrm{h}\end{array}$ & $\begin{array}{l}\text { Monitor for respiratory } \\
\text { depression, hypotension, } \\
\text { rhabdomyolysis, metabolic } \\
\text { acidosis, withdraw slowly } \\
\text { Arterial line and central access } \\
\text { recommended for high-dose } \\
\text { titration - will get hypotensive }\end{array}$ \\
\hline Pentobarbital & $\begin{array}{l}\text { GABA } \\
\text { receptor } \\
\text { agonist }\end{array}$ & $\begin{array}{l}5 \mathrm{mg} / \mathrm{kg} \\
\text { bolus, then } \\
0.5-1 \\
\mathrm{mg} / \mathrm{kg} / \mathrm{h}\end{array}$ & $\begin{array}{l}\text { Bolus } 5 \mathrm{mg} / \mathrm{kg} \\
\text { and increase by } \\
0.5 \mathrm{mg} / \mathrm{kg} / \mathrm{h} \text { every } \\
15-20 \mathrm{~min} \text { until } \\
\text { burst suppression }\end{array}$ & $\begin{array}{l}0.5-3 \\
\mathrm{mg} / \mathrm{kg} / \mathrm{h}\end{array}$ & $\begin{array}{l}10 \mathrm{mg} / \mathrm{kg} / \mathrm{h} \text { (brief } \\
\text { duration only) }\end{array}$ & $\begin{array}{l}\text { Monitor for propylene glycol } \\
\text { toxicity - dose and duration } \\
\text { dependent (serum Osm and Osm } \\
\text { gap, } \mathrm{pH}, \text { lactic acid, BUN, } \mathrm{SCr} \\
\text { and EKG) } \\
\text { Arterial line and central access } \\
\text { strongly recommended - will get } \\
\text { hypotensive }\end{array}$ \\
\hline Propofol & $\begin{array}{l}\text { GABA } \\
\text { receptor } \\
\text { agonist }\end{array}$ & $\begin{array}{l}2.5-3.5 \\
\mathrm{mg} / \mathrm{kg} \text { bolus, } \\
\text { then } 25-100 \\
\mathrm{mcg} / \mathrm{kg} / \mathrm{min}\end{array}$ & $\begin{array}{l}\text { Bolus } 3 \mathrm{mg} / \mathrm{kg} \text { and } \\
\text { increase by } 25 \\
\mathrm{mcg} / \mathrm{kg} / \mathrm{min} \text { every } \\
15-20 \mathrm{~min} \text { until } \\
\text { burst suppression }\end{array}$ & $\begin{array}{l}15-200 \\
\mathrm{mcg} / \mathrm{kg} / \mathrm{min} \\
\mathrm{Max} \text { dose } \\
4 \mathrm{mg} / \mathrm{kg} / \mathrm{h} \\
\text { per package } \\
\text { insert }\end{array}$ & $300 \mathrm{mcg} / \mathrm{kg} / \mathrm{min}$ & $\begin{array}{l}\text { Requires propofol consent before } \\
\text { use, Monitor BP, } \\
\text { Monitor triglycerides, } \\
\text { pancreatitis, PRIS; Calculate } \\
\text { lipid load from propofol into } \\
\text { nutrition calculations; } \\
\text { Arterial line and central access } \\
\text { strongly recommended - will get } \\
\text { hypotensive }\end{array}$ \\
\hline Ketamine & $\begin{array}{l}\text { NMDA } \\
\text { receptor } \\
\text { antagonist }\end{array}$ & $\begin{array}{l}0.5-1 \\
\mathrm{mg} / \mathrm{kg} / \mathrm{h}\end{array}$ & $\begin{array}{l}\text { Bolus } 0.5 \mathrm{mg} / \mathrm{kg} \\
\text { and increase dose } \\
\text { by } 0.5 \mathrm{mg} / \mathrm{kg} / \mathrm{h} \\
\text { every } 15-20 \mathrm{~min} \\
\text { until burst } \\
\text { suppression }\end{array}$ & $2-3 \mathrm{mg} / \mathrm{kg} / \mathrm{h}$ & $7.5 \mathrm{mg} / \mathrm{kg} / \mathrm{h}$ & $\begin{array}{l}\text { Co-administer with benzo- } \\
\text { diazepine (or barbiturate) to } \\
\text { prevent dissociative syndrome; } \\
\text { Monitor BP, HR (typically see } \\
\text { increases on initiation) }\end{array}$ \\
\hline
\end{tabular}

of hypotension [50]. One of the important confounding factors in this study was that the target of treatment in patients receiving pentobarbital tended to be burstsuppression, while it was limited to seizure suppression in those treated with MDZ and propofol. In pediatric RSE/ SRSE the efficacy of pentobarbital has been reported to be $74-100 \%$ in heterogeneous studies [47,51]. Some experts believe that barbiturates have a neuroprotective effect and have an additional anti-seizure efficacy from their ability to lower core body temperature [52]. However, barbiturates have more potent cardiorespiratory suppression, immunosuppression, longer half-life with storage in the lipid compartment resulting in slow recovery, and problems of auto-induction and zero-order kinetics. Intravenous formulations of pentobarbital also contain propylene glycol, which at the high cumulative doses necessary to achieve burst suppression, may cause hyperosmolality and lactic acidosis that can progress to cardiac arrhythmias, refractory hypotension, renal dysfunction, and multi-organ failure [53]. It is possible that advances in intensive care, and the increasing practice for pre-emptive control of ventilation and perfusion, may help mitigate some of these challenges.

In adults, propofol is used for management of RSE/ SRSE due to its quick onset of action and prompt recovery on withdrawal. Studies in adults have shown that propofol infusion terminates RSE/SRSE in $67 \%$ of patients [54]. Although propofol induces burst-suppression within 35 minutes of initiation, frequent titration may be needed to maintain adequate suppression [55]. A retrospective study of 33 children aged 4 months to 15 years with RSE reported that propofol was more effective than thiopental in terminating seizures (64\% vs 55\%). However, adverse effects including rhabdomyolysis and hypertriglyceridemia, prompted discontinuation in $18 \%$ of patients, with recovery after discontinuation of propofol [56]. Use of propofol has been restricted in children due to the risk of 
propofol infusion syndrome (cardiac failure, rhabdomyolysis, metabolic acidosis, renal failure, and sometimes death) and propensity to induce dyskinesias which can mimic breakthrough seizures [57]. Risk factors reported to be associated with a higher likelihood of the infusion syndrome include high doses, prolonged use, concurrent use of catecholamines and corticosteroids, and possibly malnutrition. However, there have been some concerns that this syndrome may not be limited to propofol, and could in fact, result from drug-induced cerebral suppression [58]. Also relevant to pediatric practice are the reported fatalities on concurrent use of propofol and ketogenic diet (KD) [59].

\section{Ketamine}

Acceptance for the use of ketamine in RSE/SRSE has been steadily increasing due to favorable hemodynamics and a different mechanism of action than conventional anesthetics. Given the GABA receptor endocytosis in RSE/SRSE, and the NMDA glutamate receptor antagonism of ketamine, there is a theoretical advantage for its use instead of, or more commonly in addition to, other anesthetic agents. A systematic review of 25 class IV studies analyzed 244 SE episodes (37 in children) treated with ketamine [60]. Although authors reported $73 \%$ of children to 'respond', the heterogeneity in the definition of response was not addressed. Our practice is to start with MDZ infusion, and optimize it to achieve seizure suppression and $>50 \%$ burst suppression. In nonresponders, we then consider ketamine concurrent to MDZ. In patients who do not respond, we finally start pentobarbital and wean off MDZ and ketamine (Fig. 1). This is different from published multicenter experience from US, where ketamine was typically used after pentobarbital [61]. Salient pharmacological aspects of these drugs are summarized in Table III.

\section{Therapeutic Target for RSE}

As we have already alluded, there is a lack of consensus regarding optimal target for treatment of RSE/SRSE. Different therapeutic endpoints have included seizure suppression, burst suppression, or electro-cerebral silence. Moreover, the extent of burst-suppression that is associated with best outcomes, remains undefined [62]. This results in variability in clinical practice as shown by a study including 35 adult RSE patients, which reported that patients remained within the target suppression range (defined as $65-95 \%$ ) only $0-29 \%$ (median $8 \%$ ) of the total time under treatment [63]. Our practice is to increase infusions rapidly to achieve a suppression ratio of $50-70 \%$ and complete seizure control, as soon as possible after failure of $2^{\text {nd }}$ line treatment, and to frequently review EEG to ensure that this degree of suppression is maintained.
We try to wean off anesthetic infusions once this treatment target is maintained for 24-48 hours. However, this duration is not based on rigorous evidence, and a shorter duration of burst suppression may be beneficial in some patients, whereas several cycles may be needed in patients with SRSE. Longer pharmacological coma of up to 5-7 days may have to be tolerated in particularly challenging cases. Simultaneously, additional ASMs preferably with complimentary mechanisms of action, short half-life, and low incidence of drug inter-actions, should be added to facilitate ongoing seizure suppression and smooth weaning of infusions. Topira-mate, LEV, LAC, and more recently, brivaracetam and perampanel have been used for this purpose [26].

The effectiveness of this empirical practice was recently assessed in a cohort of 111 RSE patients recruited prospectively over two years in an observational study. MDZ was the most frequently used initial anesthetic agent (78\%), and pentobarbital was most frequently used agent after MDZ failure (82\%) [64]. Treating physicians used up to four cycles of serial anesthetic therapy in these patients, and seizure termination was achieved in $94 \%$ patients by the second cycle. However, other studies have shown regional differences in the use of therapeutic coma for RSE treatment, lack of effect on overall mortality, and increased length of hospital stay, related costs, and adverse effects $[65,66]$.

A suggested protocol for management of RSE/SRSE, based on the practice at authors' institutions is provided as Fig. 1. This protocol is applicable only to patients $>29$ days of age. Given the lack of class I evidence, modifications to this protocol driven by local epidemiology and resources are strongly encouraged. Treatments other than anesthetic infusions may be considered earlier based on suspected/proven etiology of status epilepticus.

\section{Additional Treatment Options}

Patients with RSE, particularly after failure of first anesthetic infusion, or those with SRSE, represent a desperate situation for the clinical team. Hence, myriad approaches are brought upon to control the RSE/SRSE, often based on limited experience. These diverse treatments have been discussed elsewhere [67]. The relative position of these modalities in the treatment of SRSE has to be individualized according to the patient and the clinical resources.

\section{Ketogenic Diet}

Among these approaches, the most promising appears to be ketogenic diet (KD), partly due to its potential for undermining pathophysiology of RSE/SRSE [68,69]. A 
IV midazolam:

- Give $0.2 \mathrm{mg} / \mathrm{kg}$ bolus (maximum $10 \mathrm{mg}$ ), then start infusion at $0.2 \mathrm{mg} / \mathrm{kg} / \mathrm{h}$ (maximum $10 \mathrm{mg} / \mathrm{h}$ )

- Increase infusion rate by $0.2 \mathrm{mg} / \mathrm{kg} / \mathrm{h}$ (maximum $10 \mathrm{mg} / \mathrm{h}$ ) every 10 minutes until target burst suppression or reach a dose of $2 \mathrm{mg} / \mathrm{kg} / \mathrm{h}$ (maximum $100 \mathrm{mg} / \mathrm{h}$ )

- Prepare to add IV ketamine when midazolam infusion reaches $1.6 \mathrm{mg} / \mathrm{kg} / \mathrm{h}$

- Perform endotracheal intubation if not already done

- Start continuous EEG monitoring

- Transfer to pediatric ICU

Therapeutic target not achieved

Add IV ketamine:

- Start infusion at $0.5 \mathrm{mg} / \mathrm{kg} / \mathrm{h}$

- Increase infusion rate by $0.5 \mathrm{mg} / \mathrm{kg} / \mathrm{h}$ every 15 20 minutes until target burst suppression or maximum dose of $7.5 \mathrm{mg} / \mathrm{kg} / \mathrm{h}$

- Prepare to add IV pentobarbital infusion when IV ketamine infusion reaches $6 \mathrm{mg} / \mathrm{kg} / \mathrm{h}$

Therapeutic target not achieved

$\downarrow$

IV pentobarbital:

- Give $5 \mathrm{mg} / \mathrm{kg}$ bolus, then start infusion at $1 \mathrm{mg} /$ $\mathrm{kg} / \mathrm{h}$

- Increase infusion rate by $0.5 \mathrm{mg} / \mathrm{kg} / \mathrm{h}$ every 15 20 minutes until target burst suppression or maximum dose of $3 \mathrm{mg} / \mathrm{kg} / \mathrm{h}$

- Decrease midazolam infusion rate to $1 \mathrm{mg} / \mathrm{kg} / \mathrm{h}$ at the start of pentobarbital infusion, stop MDZ after first increase of pentobarbital infusion to 1.5 $\mathrm{mg} / \mathrm{kg} / \mathrm{h}$

- Consider decreasing ketamine infusion rate also at this point

Therapeutic target not achieved

Consider other therapies (see text for details):

- Ketogenic diet

- Immunotherapy (corticosteroids, IVIg, plasmapheresis)

- Pyridoxine (if not already tried)

- Epilepsy surgery consultation
Therapeutic target achieved

Target of treatment:

- Complete suppression of seizures

- EEG burst suppression around $70 \%$ (never $<50 \%$ )

Maintain burst suppression:

- Initial: 24-48 hours

- Repeated: 48-72 hours

Weaning continuous infusions:

- $\leq 48$ hours duration: wean over 6-12 hours, decrease rate by $15-30 \%$ every 2 hours

- $>48$ hours duration: slow wean, decrease rate by $15-30 \%$ every $6-12$ hours

- Consider adding scheduled benzodiazepines or barbiturates for withdrawal for infusions $>5$ days

Add maintenance anti-seizure medications:

- Use doses at high end of therapeutic range

- Consider combinations with multiple different mechanisms

Fig.1 A suggested protocol for management of refractory/super-refractory status epilepticus. 
study including 14 pediatric patients reported electrographic seizure resolution and $\geq 50 \%$ suppression in 10 patients within 7 days of starting KD [70]. In 11/14 patients, continuous infusions could be weaned off within two weeks of starting KD. However, the authors noted under-utilization of and delay in starting KD. Other series with 8-17 patients have also reported seizure resolution within 7 days of starting KD in 20-90\% patients $[67,70]$. KD is typically administered via enteral route, though ketogenic parenteral nutrition has been used in some cases, both in 4:1 ratio. However, ketosis may sometimes be difficult to achieve with concomitant barbiturate infusions which contain propylene glycol that is metabolized to lactaldehyde and then to lactic acid. Common reported adverse effects of KD have included metabolic derangements like hypoglycemia; and gastrointestinal symptoms like emesis.

\section{Surgery}

In patients with a potentially epileptogenic brain lesion and concordant neurophysiology, an urgent epilepsy surgery may be considered, if such expertise is readily available. However, such decisions also incorporate the location, size, and nature of MRI lesion(s); and functional significance of surrounding cortex, which may require intracranial EEG evaluation and electrical stimulation mapping. Surgical decision-making becomes more challenging in MRI-negative RSE/SRSE or discordant neurophysiological data. Functional imaging is often confounded in such cases by ongoing ictal activity and concurrent anesthetic infusions. In some cases, palliative options like vagus nerve stimulation, or corpus callosotomy may be considered. Non-invasive neuro-stimulation, particularly with transcranial magnetic stimulation, are emerging modalities for interrupting RSE/SRSE, which may be useful in future.

\section{Other Approaches}

Among other modalities, therapeutic hypothermia is the only intervention tested in a randomized controlled trial for RSE/SRSE management. However, such trials, including the HYBERNATUS study, found the efficacy of hypothermia to be no better than placebo for RSE/ SRSE, and raised concerns about its safety [67,71]. Immunotherapy (including any combination of steroids, intravenous immunoglobulins, or plasma exchange) may be helpful in known autoimmune epilepsies or entities with presumed immunological basis, such as febrileinfection related epilepsy syndrome (FIRES). At present, there is insufficient evidence for or against the use of immunotherapy in other RSE/SRSE patients. Similarly, there is no evidence for use of magnesium and pyridoxine outside of specific indications. These specific scenarios include using magnesium for acute convulsive seizures in eclampsia, and pyridoxine for suspected functional antiquitin deficiency or isoniazid toxicity.

\section{Inhalational Anesthetics}

There is limited experience for the use of inhaled anesthetics, primarily isoflurane and dexflurane, late in SRSE unresponsive to MDZ, propofol, and pentobarbital. In published cases, both isoflurane and dexflurane were effective in stopping seizures and achieving burst suppression; however, seizures recurred frequently after discontinuation. Complications of isoflurane and dexflurane have included hypotension, atelectasis, infections, paralytic ileus, and deep vein thrombosis; with death in 3/7 patients in one series [72]. In a recent case report, two patients with prolonged isoflurane use showed MRI changes in the thalamus and cerebellum, raising concerns for neurotoxicity [73]. More frequent hippocampal changes on MRI were seen in patients receiving isoflurane for RSE compared to matched controls that received only intravenous anesthetics; with these changes being related to longer duration of isoflurane use [74]. Given the logistical difficulties and high incidence of complications with prolonged use of inhalational anesthetics, the risk/ benefit should be carefully considered before pursuing this course of therapy.

\section{CONSEQUENCES OF RSE/SRSE}

The adverse consequences of RSE/SRSE can be divided into immediate complications and longer term neuromorbidity and mortality (Web Table I). The interim complications mainly result from aberrant pathophysiological processes that are often initiated in an attempt to control the prolonged seizure but may fail or get out-ofhand and result in neuronal injury. Additionally, patients with RSE/SRSE also face complications resulting from its treatment and those from prolonged intensive care, as summarized in Web Table 1. More protracted consequences of RSE/SRSE are conventionally classified into short-term (during hospitalization or within 30 days of onset of SE) and long-term (within 10 years following initial survival 30 days after SE onset).

In a multicenter Canadian study of 374 patients with newly diagnosed epilepsy, occurrence of convulsive SE in 22 children was associated with poor health-related quality of life after 24 months follow-up [75]. In general, RSE/SRSE have significantly higher morbidity and mortality compared to SE of shorter duration. In children, RSE is associated with mortality in $11 \%-44 \%$ of patients and long-term neurological deficits in $25 \%-100 \%$ of the survivors [76-79]. The fatality of convulsive SE was 11\% 
in the north London study, with cumulative incidence of epilepsy, intellectual disability, and motor impairment of $25 \%, 12 \%$, and $5 \%$, respectively in survivors after a median follow-up of 9 years $[78,79]$. This study also reported motor and intellectual disability to be more prevalent in patients with pre-existing epilepsy and neurologic disability. Other relatively smaller studies of pediatric RSE have reported mortality from $16 \%$ to $44 \%$, and sequelae in $25 \%-100 \%$ of survivors $[47,76,80]$. Neurological sequelae of RSE/SRSE appear to have relatively higher incidence in infants. In a large prospective study with mean follow-up of 13.2 months, the incidence of neurological deficits attributable to convulsive SE was $29 \%$ in infants $\leq 1$ year of age, $11 \%$ in children 1-3 years of age, and $6 \%$ in children $>3$ years of age [81].

The risk of subsequent development of epilepsy is $25 \%-40 \%$ within 2 years after an RSE/SRSE episode, with a higher propensity in those with symptomatic causes [82]. The risk of recurrent SE is estimated to be about $20 \%$ within 4 years of the first RSE episode, with the highest risk in the first year. Progressive and remote symptomatic etiologies are associated with a higher risk for recurrent SE compared to febrile SE or acute symptomatic SE [83]. Long term adverse behavioral outcomes are also prevalent. After a mean 8 years of follow-up in the North London cohort, 37\% SE patients were noted to have behavioral issues and $28 \%$ received a psychiatric diagnosis such as autism, attention deficit hyperactivity disorder, pervasive developmental disorder not otherwise specified, and developmental coordination disorder [84].

Several studies have reported age and etiology to be major determinants of both short-term and long-term mortality in SE [4,85]. A systematic review found mortality to be lower in children as compared to adults and elderly, with short-term mortality up to $9 \%$ and longterm mortality up to 7\% [4]. Amongst children, infants $\leq 1$ year of age had the highest short-term mortality, up to $18 \%$ [4]. Most SE-related deaths in the short term have been noted to occur in children with acute symptomatic etiology [85]. In a cohort study from Kenya, 23\% of children with confirmed CSE died before discharge, with $75 \%$ of the deaths occurring within 48 hours of onset of SE[86].

\section{Avenues for Future Research}

RSE/SRSE presents a host of important unanswered clinical conundrums which have significant potential to impact patient care. Perhaps the foremost among these is to generate randomized, blinded, and adequately controlled evidence for various treatment regimens.
Secondly, the optimal target for treatment needs to be defined. This will require a careful evaluation of EEG biomarkers for outcomes of RSE/SRSE. Thirdly, it is desirable to develop multicenter registries of RSE/SRSE with longitudinal data, which can be harnessed for predictive modelling of outcomes. There are unique challenges and opportunities in this regard for pediatricians and neurologists in India. There is a need to generate data about epidemiology, causes, diagnostic, and therapeutic modalities and their yield in India to develop targeted strategies for intervention that are specific to local circumstances.

\section{REFERENCES}

1. Sanchez Fernandez I, Abend NS, Agadi S, An S, Arya R, Carpenter JL, et al. Gaps and opportunities in refractory status epilepticus research in children: A multi-center approach by the Pediatric Status Epilepticus Research Group (pSERG). Seizure. 2014;23:87-97.

2. Shorvon S, Ferlisi M. The treatment of super-refractory status epilepticus: A critical review of available therapies and a clinical treatment protocol. Brain. 2011;134:280218.

3. Mayer SA, Claassen J, Lokin J, Mendelsohn F, Dennis LJ, Fitzsimmons BF. Refractory status epilepticus: frequency, risk factors, and impact on outcome. Arch Neurol. 2002;59: 205-10.

4. Chin RF, Neville BG, Scott RC. A systematic review of the epidemiology of status epilepticus. Eur J Neurol. 2004;11:800-10.

5. Shinnar S, Bello JA, Chan S, Hesdorffer DC, Lewis DV, Macfall J, et al. MRI abnormalities following febrile status epilepticus in children: the FEBSTAT study. Neurology. 2012;79: 871-7.

6. Lewis DV, Shinnar S, Hesdorffer DC, Bagiella E, Bello JA, Chan $\mathrm{S}$, et al. Hippocampal sclerosis after febrile status epilepticus: the FEBSTAT study. Ann Neurol. 2014;75:178-85.

7. Hesdorffer DC, Shinnar S, Lewis DV, Nordli DR, Jr., Pellock JM, Moshe SL, et al. Risk factors for febrile status epilepticus: a case-control study. J Pediatr. 2013;163:1147-51.

8. Bassan H, Barzilay M, Shinnar S, Shorer Z, Matoth I, Gross-Tsur V. Prolonged febrile seizures, clinical characteristics, and acute management. Epilepsia. 2013;54:1092-8.

9. Gulati S, Kalra V, Sridhar MR. Status epilepticus in Indian children in a tertiary care center. Indian $\mathrm{J}$ Pediatr. 2005;72:105-8.

10. Sasidaran K, Singhi S, Singhi P. Management of acute seizure and status epilepticus in pediatric emergency. Indian J Pediatr. 2012;79:510-7.

11. Arya R, Gulati S, Kabra M, Sahu JK, Kalra V. Intranasal versus intravenous lorazepam for control of acute seizures in children: A randomized open-label study. Epilepsia. 2011;52:788-93.

12. Misra UK, Kalita J. Seizures in encephalitis: predictors and 
outcome. Seizure. 2009;18:583-7.

13. Whitley RJ, Alford CA, Hirsch MS, Schooley RT, Luby JP, Aoki FY, et al. Vidarabine versus acyclovir therapy in herpes simplex encephalitis. N Engl J Med. 1986;314:1449.

14. Murthy JMK, Deshmukh DS. Convulsive status epilepticus due to different evolutionary stages of neurocysticercosis solitary cyticercus granuloma, low cyst load, and single calcific lesion in an endemic country: Clinical profile. Seizure. 2019;71:229-32.

15. Graus F, Titulaer MJ, Balu R, Benseler S, Bien CG, Cellucci $\mathrm{T}$, et al. A clinical approach to diagnosis of autoimmune encephalitis. Lancet Neurol. 2016;15:391-404.

16. Armangue T, Petit-Pedrol M, Dalmau J. Autoimmune encephalitis in children. J Child Neurol. 2012;27:1460-9.

17. Machado S, Pinto AN, Irani SR. What should you know about limbic encephalitis? Arq Neuropsiquiatr. 2012;70:817-22.

18. Bhatnagar M, Shorvon S. Genetic mutations associated with status epilepticus. Epilepsy Behav. 2015;49:104-10.

19. Brophy GM, Bell R, Claassen J, Alldredge B, Bleck TP, Glauser $\mathrm{T}$, et al. Guidelines for the evaluation and management of status epilepticus. Neurocrit Care. 2012;17:3-23.

20. Herman ST, Abend NS, Bleck TP, Chapman KE, Drislane FW, Emerson RG, et al. Consensus statement on continuous EEG in critically ill adults and children, part I: Indications. J Clin Neurophysiol. 2015;32:87-95.

21. Herman ST, Abend NS, Bleck TP, Chapman KE, Drislane $\mathrm{FW}$, Emerson RG, et al. Consensus statement on continuous EEG in critically ill adults and children, part II: Personnel, technical specifications, and clinical practice. J Clin Neurophysiol. 2015;32:96-108.

22. Leitinger M, Trinka E, Gardella E, Rohracher A, Kalss G, Qerama E, et al. Diagnostic accuracy of the Salzburg EEG criteria for non-convulsive status epilepticus: A retrospective study. Lancet Neurol. 2016;15:1054-62.

23. Beniczky S, Hirsch LJ, Kaplan PW, Pressler R, Bauer G, Aurlien $\mathrm{H}$, et al. Unified EEG terminology and criteria for nonconvulsive status epilepticus. Epilepsia. 2013;54:28-9.

24. Hirsch LJ, LaRoche SM, Gaspard N, Gerard E, Svoronos A, Herman ST, et al. American Clinical Neurophysiology Society's Standardized Critical Care EEG Terminology: 2012 version. J Clin Neurophysiol. 2013;30:1-27.

25. Sansevere AJ, Hahn CD, Abend NS. Conventional and quantitative EEG in status epilepticus. Seizure. 2019;68:38-45.

26. Ferlisi M, Shorvon S. The outcome of therapies in refractory and super-refractory convulsive status epilepticus and recommendations for therapy. Brain. 2012;135: 2314-28.

27. Pearl PL, Vezina LG, Saneto RP, McCarter R, MolloyWells E, Heffron A, et al. Cerebral MRI abnormalities associated with vigabatrin therapy. Epilepsia 2009;50: 184-94.

28. Milh M, Villeneuve N, Chapon F, Pineau S, Lamoureux S, Livet $\mathrm{MO}$, et al. Transient brain magnetic resonance imaging hyperintensity in basal ganglia and brain stem of epileptic infants treated with vigabatrin. J Child Neurol.
2009;24: 305-15.

29. Loscher W. Mechanisms of drug resistance in status epilepticus. Epilepsia. 2007;48:74-7.

30. Hocker S. Systemic complications of status epilepticusAn update. Epilepsy Behav. 2015;49:83-7.

31. Glauser T, Shinnar S, Gloss D, Alldredge B, Arya R, Bainbridge J, et al. Evidence-Based Guideline: Treatment of Convulsive Status Epilepticus in Children and Adults: Report of the Guideline Committee of the American Epilepsy Society. Epilepsy Curr. 2016;16:48-61.

32. Treiman DM, Meyers PD, Walton NY, Collins JF, Colling $\mathrm{C}$, Rowan AJ, et al. A comparison of four treatments for generalized convulsive status epilepticus. Veterans Affairs Status Epilepticus Cooperative Study Group. N Engl J Med. 1998;339:792-8.

33. Silbergleit R, Durkalski V, Lowenstein D, Conwit R, Pancioli A, Palesch Y, et al. Intramuscular versus intravenous therapy for prehospital status epilepticus. N Engl J Med. 2012;366:591-600.

34. Chamberlain JM, Okada P, Holsti M, Mahajan P, Brown $\mathrm{KM}$, Vance $\mathrm{C}$, et al. Lorazepam vs diazepam for pediatric status epilepticus: a randomized clinical trial. JAMA. 2014;311:1652-60.

35. Arya R, Kothari H, Zhang Z, Han B, Horn PS, Glauser TA. Efficacy of nonvenous medications for acute convulsive seizures: A network meta-analysis. Neurology. 2015;85:1859-68.

36. US Food and Drug Administrations. https://www. accessdata.fda.gov/drugsatfda_docs/label/2019/211321s 000lbl .pdf. Accessed July 31, 2019.

37. Neuralis Inc. Our pipeline. https://www.neurelis.com/ourpipeline/valtoco-nasal-spray. Accessed July 31, 2019.

38. French JA, Wechsler R, Gelfand MA, Pollard JR, Vazquez $\mathrm{B}$, Friedman $\mathrm{D}$, et al. Inhaled alprazolam rapidly suppresses epileptic activity in photosensitive participants. Epilepsia. 2019.

39. Yasiry Z, Shorvon SD. The relative effectiveness of five antiepileptic drugs in treatment of benzodiazepine-resistant convulsive status epilepticus: A meta-analysis of published studies. Seizure. 2014;23:167-74.

40. Dalziel SR, Borland ML, Furyk J, Bonisch M, Neutze J, Donath $\mathrm{S}$, et al. Levetiracetam versus phenytoin for second-line treatment of convulsive status epilepticus in children (ConSEPT): An open-label, multicentre, randomised controlled trial. Lancet. 2019;393:2135-45.

41. Lyttle MD, Rainford NEA, Gamble C, Messahel S, Humphreys A, Hickey $\mathrm{H}$, et al. Levetiracetam versus phenytoin for second-line treatment of paediatric convulsive status epilepticus (EcLiPSE): A multicentre, open-label, randomised trial. Lancet. 2019;393:2125-34.

42. Bleck T, Cock H, Chamberlain J, Cloyd J, Connor J, Elm J, et al. The established status epilepticus trial 2013. Epilepsia. 2013;54:89-92.

43. Rossetti AO, Milligan TA, Vulliemoz S, Michaelides C, Bertschi M, Lee JW. A randomized trial for the treatment of refractory status epilepticus. Neurocrit Care. 2011;14:4-10.

44. Rosenthal ES, Claassen J, Wainwright MS, Husain AM, Vaitkevicius $\mathrm{H}$, Raines $\mathrm{S}$, et al. Brexanolone as adjunctive therapy in super-refractory status epilepticus. Ann Neurol. 
2017;82:342-52.

45. Abend NS, Dlugos DJ. Treatment of refractory status epilepticus: Literature review and a proposed protocol. Pediatr Neurol. 2008;38:377-90.

46. Naritoku DK, Sinha S. Prolongation of midazolam half-life after sustained infusion for status epilepticus. Neurology. 2000;54:1366-8.

47. Gilbert DL, Gartside PS, Glauser TA. Efficacy and mortality in treatment of refractory generalized convulsive status epilepticus in children: A meta-analysis. J Child Neurol. 1999;14:602-9.

48. Singhi S, Murthy A, Singhi P, Jayashree M. Continuous midazolam versus diazepam infusion for refractory convulsive status epilepticus. J Child Neurol. 2002; 17:106-10.

49. Morrison G, Gibbons E, Whitehouse WP. High-dose midazolam therapy for refractory status epilepticus in children. Intensive Care Med. 2006;32:2070-6.

50. Claassen J, Hirsch LJ, Emerson RG, Mayer SA. Treatment of refractory status epilepticus with pentobarbital, propofol, or midazolam: A systematic review. Epilepsia. 2002;43:146-53.

51. Kim SJ, Lee DY, Kim JS. Neurologic outcomes of pediatric epileptic patients with pentobarbital coma. Pediatr Neurol. 2001;25:217-20.

52. Holtkamp M, Masuhr F, Harms L, Einhaupl KM, Meierkord H, Buchheim K. The management of refractory generalised convulsive and complex partial status epilepticus in three European countries: A survey among epileptologists and critical care neurologists. J Neurol Neurosurg Psychiatry. 2003;74:1095-9.

53. Bledsoe KA, Kramer AH. Propylene glycol toxicity complicating use of barbiturate coma. Neurocrit Care. 2008;9:122-4

54. Rossetti AO, Reichhart MD, Schaller MD, Despland PA, Bogousslavsky J. Propofol treatment of refractory status epilepticus: A study of 31 episodes. Epilepsia. 2004;45:757-63.

55. Parviainen I, Uusaro A, Kalviainen R, Mervaala E, Ruokonen E. Propofol in the treatment of refractory status epilepticus. Intensive Care Med. 2006;32:1075-9.

56. van Gestel JP, Blusse van Oud-Alblas HJ, Malingre M, Ververs FF, Braun KP, van Nieuwenhuizen O. Propofol and thiopental for refractory status epilepticus in children. Neurology. 2005;65:591-2.

57. McHugh P. Acute choreoathetoid reaction to propofol. Anaesthesia. 1991;46: 425.

58. Enting D, Ligtenberg JJ, Aarts LP, Zijlstra JG. Total suppression of cerebral activity by thiopental mimicking propofol infusion syndrome: A fatal common pathway? Anesth Analg. 2005;100:1864-5; author reply 1865.

59. Baumeister FA, Oberhoffer R, Liebhaber GM, Kunkel J, Eberhardt J, Holthausen H, Peters J. Fatal propofol infusion syndrome in association with ketogenic diet. Neuropediatrics. 2004;35:250-2.

60. Hofler J, Trinka E. Intravenous ketamine in status epilepticus. Epilepsia. 2018;59: 198-206.

61. Keros S, Buraniqi E, Alex B, Antonetty A, Fialho H, Hafeez $\mathrm{B}$, et al. Increasing ketamine use for refractory status epilepticus in US pediatric hospitals. J Child Neurol. 2017;32: 638-46.

62. Rossetti AO, Logroscino G, Bromfield EB. Refractory status epilepticus: Effect of treatment aggressiveness on prognosis. Arch Neurol. 2005;62:1698-702.

63. An J, Jonnalagadda D, Moura VJ, Purdon PL, Brown EN, Westover MB. Variability in pharmacologically-induced coma for treatment of refractory status epilepticus. PLoS. One 2018;13:e0205789.

64. Tasker RC, Goodkin HP, Sanchez Fernandez I, Chapman KE, Abend NS, Arya R, et al. Refractory status epilepticus in children: Intention to treat with continuous infusions of midazolam and pentobarbital. Pediatr Crit Care Med. 2016;17:968-75.

65. Alvarez V, Lee JW, Westover MB, Drislane FW, Novy J, Faouzi M, et al. Therapeutic coma for status epilepticus: Differing practices in a prospective multicenter study. Neurology. 2016;87:1650-9.

66. Sutter R, Marsch S, Fuhr P, Kaplan PW, Ruegg S. Anesthetic drugs in status epilepticus: risk or rescue? A 6year cohort study. Neurology. 2014;82:656-64.

67. Arya R, Rotenberg A. Dietary, immunological, surgical, and other emerging treatments for pediatric refractory status epilepticus. Seizure. 2019;68:89-96.

68. Likhodii SS, Serbanescu I, Cortez MA, Murphy P, Snead OC, 3rd, Burnham WM. Anticonvulsant properties of acetone, a brain ketone elevated by the ketogenic diet. Ann Neurol. 2003;54:219-26.

69. Rho JM, Anderson GD, Donevan SD, White HS. Acetoacetate, acetone, and dibenzylamine (a contaminant in 1-(+)-beta-hydroxybutyrate) exhibit direct anticonvulsant actions in vivo. Epilepsia. 2002;43:358-61.

70. Arya R, Peariso K, Gainza-Lein M, Harvey J, Bergin A, Brenton JN, et al. Efficacy and safety of ketogenic diet for treatment of pediatric convulsive refractory status epilepticus. Epilepsy Res. 2018;144:1-6.

71. Legriel S, Lemiale V, Schenck M, Chelly J, Laurent V, Daviaud F, et al. Hypothermia for neuroprotection in convulsive status epilepticus. N Engl J Med. 2016;375:2457-67.

72. Mirsattari SM, Sharpe MD, Young GB. Treatment of refractory status epilepticus with inhalational anesthetic agents isoflurane and desflurane. Arch Neurol. 2004;61:1254-9.

73. Fugate JE, Burns JD, Wijdicks EF, Warner DO, Jankowski CJ, Rabinstein AA. Prolonged high-dose isoflurane for refractory status epilepticus: is it safe? Anesth Analg. 2010;111:1520-4.

74. Ikeda KM, Connors R, Lee DH, Khandji AG, Claassen J, Young GB. Isoflurane use in the treatment of superrefractory status epilepticus is associated with hippocampal changes on MRI. Neurocrit Care. 2017;26:420-7.

75. Ferro MA, Chin RF, Camfield CS, Wiebe S, Levin SD, Speechley KN. Convulsive status epilepticus and healthrelated quality of life in children with epilepsy. Neurology. 2014;83:752-7.

76. Sahin M, Menache CC, Holmes GL, Riviello JJ. Outcome of severe refractory status epilepticus in children. Epilepsia. 2001;42:1461-7. 
77. Neligan A, Shorvon SD. Frequency and prognosis of convulsive status epilepticus of different causes: A systematic review. Arch Neurol. 2010;67:931-40.

78. Pujar SS, Neville BG, Scott RC, Chin RF, North London Epilepsy Research N. Death within 8 years after childhood convulsive status epilepticus: a population-based study. Brain. 2011;134:2819-27.

79. Pujar SS, Martinos MM, Cortina-Borja M, Chong WKK, De Haan M, Gillberg C, et al. Long-term prognosis after childhood convulsive status epilepticus: A prospective cohort study. Lancet Child Adolesc Health. 2018;2: 103-111.

80. Sahin M, Menache CC, Holmes GL, Riviello JJ, Jr. Prolonged treatment for acute symptomatic refractory status epilepticus: outcome in children. Neurology. 2003;61:398-401.

81. Maytal J, Shinnar S, Moshe SL, Alvarez LA. Low morbidity and mortality of status epilepticus in children. Pediatrics. 1989;83:323-31.
82. Gurcharran K, Grinspan ZM. The burden of pediatric status epilepticus: Epidemiology, morbidity, mortality, and costs. Seizure 2019;68:3-8.

83. Jafarpour S, Stredny CM, Piantino J, Chapman KE. Baseline and outcome assessment in pediatric status epilepticus. Seizure. 2019;68:52-61.

84. Martinos MM, Pujar S, Gillberg C, Cortina-Borja M, Neville BGR, De Haan M, et al. Long-term behavioural outcomes after paediatric convulsive status epilepticus: A population-based cohort study. Dev Med Child Neurol. 2018;60: 409-16.

85. DeLorenzo RJ, Hauser WA, Towne AR, Boggs JG, Pellock JM, Penberthy L, et al. A prospective, population-based epidemiologic study of status epilepticus in Richmond, Virginia. Neurology. 1996;46:1029-35.

86. Sadarangani M, Seaton C, Scott JA, Ogutu B, Edwards T, Prins A, et al. Incidence and outcome of convulsive status epilepticus in Kenyan children: A cohort study. Lancet Neurol. 2008;7:145-50. 